Proceedings of the Edinburgh Mathematical Society (2004) 47, 1-13 (C)

DOI:10.1017/S0013091503000105 Printed in the United Kingdom

\title{
EXISTENCE OF POSITIVE SOLUTIONS FOR BOUNDARY-VALUE PROBLEMS WITH SINGULARITIES IN PHASE VARIABLES
}

\author{
RAVI P. AGARWAL ${ }^{1}$, DONAL O'REGAN ${ }^{2}$ AND SVATOSLAV STANĚK ${ }^{3}$ \\ ${ }^{1}$ Department of Mathematical Sciences, Florida Institute of Technology, \\ Melbourne, Florida 32901-6975, USA (agarwal@fit.edu) \\ ${ }^{2}$ Department of Mathematics, National University of Ireland, \\ Galway, Ireland (donal.oregan@nuigalway.ie) \\ ${ }^{3}$ Department of Mathematical Analysis, Faculty of Science, \\ Palacký University, Tomkova 40, 77900 Olomouc, \\ Czech Republic (stanek@risc.upol.cz)
}

(Received 12 February 2003)

\begin{abstract}
The singular boundary-value problem $\left(g\left(x^{\prime}\right)\right)^{\prime}=\mu f\left(t, x, x^{\prime}\right), x^{\prime}(0)=0, x(T)=b>0$ is considered. Here $\mu$ is the parameter and $f(t, x, y)$, which satisfies local Carathéodory conditions on $[0, T] \times(\mathbb{R} \backslash\{b\}) \times(\mathbb{R} \backslash\{0\})$, may be singular at the values $x=b$ and $y=0$ of the phase variables $x$ and $y$, respectively. Conditions guaranteeing the existence of a positive solution to the above problem for suitable positive values of $\mu$ are given. The proofs are based on regularization and sequential techniques and use the topological transversality theorem.
\end{abstract}

Keywords: singular boundary-value problem; mixed condition; positive solution; topological transversality theorem

2000 Mathematics subject classification: Primary 34B16; 34B18

\section{Introduction}

Let $b, T$ be positive numbers, $J=[0, T]$ and $\mathbb{R}_{a}=\mathbb{R} \backslash\{a\}$ for $a \in[0, \infty)$. Consider the boundary-value problem (BVP)

$$
\begin{gathered}
\left(g\left(x^{\prime}(t)\right)\right)^{\prime}=\mu f\left(t, x(t), x^{\prime}(t)\right), \\
x^{\prime}(0)=0, \quad x(T)=b .
\end{gathered}
$$

Here $\mu$ is the parameter, $g \in C^{0}(\mathbb{R}), f$ satisfies local Carathéodory conditions on $J \times$ $\mathbb{R}_{b} \times \mathbb{R}_{0}\left(f \in \operatorname{Car}\left(J \times \mathbb{R}_{b} \times \mathbb{R}_{0}\right)\right)$, and $f(t, x, y)$ may be singular at the values $x=b$ and $y=0$ of the phase variable $x$ and $y$, respectively.

Together with (1.2) we also discuss the boundary conditions

$$
x^{\prime}(0)=0, \quad x(T)=c, \quad 0<c<b .
$$


A function $x \in C^{1}(J)$ is said to be a solution of the $B V P(1.1),(\mathrm{j}), j=1.2,1.3$, if $g\left(x^{\prime}\right) \in \mathrm{AC}(J)$ (absolutely continuous functions on $\left.J\right), x$ satisfies the boundary conditions (j) and (1.1) holds a.e. on $J$.

The aim of the paper is to give conditions guaranteeing the existence of positive solutions to BVPs (1.1), (j), $j=1.2,1.3$, for suitable positive values of the parameter $\mu$ in (1.1).

The study of BVPs (1.1), (1.2) and (1.1), (1.3) was motivated by the paper [4] where the results in [5] were extended and generalized. In [4] the authors discussed the existence of a non-negative solution to the singular (at the time variable $t$ ) mixed BVP

$$
\left.\begin{array}{c}
\frac{1}{p(t)}\left(p(t) x^{\prime}(t)\right)^{\prime}+\mu q(t) f_{1}\left(t, x(t), p(t) x^{\prime}(t)\right)=0, \\
\lim _{t \rightarrow 0^{+}} p(t) x^{\prime}(t)=0, \quad x(1)=b,
\end{array}\right\}
$$

with $f_{1} \in C^{0}\left([0,1] \times \mathbb{R}^{2}\right)$ satisfying $f_{1}\left(t, x, r_{0}(t)\right)=0$ for $t \in[0,1]$ and $x \in[0, \beta]$, where $r_{0} \in C^{0}([0,1])$ is positive and non-decreasing and $\beta>0$. Our Equation (1.1) is, on the one hand, a special case of (1.4) with $p=1, q=1$, but, on the other hand, it is a generalization of (1.4), since on the left-hand side of (1.1) we have $\left(g\left(x^{\prime}\right)\right)^{\prime}$ and the nonlinearity $f(t, x, y)$ may be singular at the values $x=b$ and $y=0$ of the phase variables $x$ and $y$, respectively.

Note that positive solutions to BVP (1.1), (1.2) with $g(u) \equiv u$ have been considered in $[\mathbf{2}]$. Here $f$ may be singular at the value 0 in both its phase variables and $f$ satisfies sign conditions. Conditions guaranteeing the existence of two positive solutions for the regular differential equation $\left(\left|x^{\prime}(t)\right|^{p-2} x^{\prime}(t)\right)^{\prime}+h(t) f_{2}\left(x^{\prime}(t)\right)=0, p>1$, satisfying (1.2) are given in $[\mathbf{3}]$.

Throughout this paper the following assumptions are satisfied.

$\left(H_{1}\right) g \in C^{0}(\mathbb{R})$ is increasing on $[0, \infty), g(0)=0$ and $\lim _{u \rightarrow \infty} g(u)=\infty$.

$\left(H_{2}\right) f \in \operatorname{Car}\left(J \times \mathbb{R}_{b} \times \mathbb{R}_{0}\right)$,

$$
f(t, x, \alpha(t))=0 \quad \text { for a.e. } t \in J \text { and each } x \in[0, b),
$$

where $\alpha \in C^{0}(J)$ is positive and non-decreasing on $J$ and also there are positive numbers $\varepsilon, \varepsilon_{0}$ and $\nu \in(0, T]$ such that

$$
\varepsilon \leqslant f(t, x, y) \text { for a.e. } t \in[0, \nu] \text { and each }(x, y) \in[0, b) \times\left(0, \varepsilon_{0}\right] .
$$

$\left(H_{3}\right)$ For a.e. $t \in J$ and each $(x, y) \in[0, b) \times(0, \alpha(t)]$,

$$
0 \leqslant f(t, x, y) \leqslant\left(q_{1}(x)+q_{2}(x)\right)\left(\omega_{1}(y)+\omega_{2}(y)\right),
$$

where $q_{2} \in C^{0}([0, b)), \omega_{1} \in C^{0}([0, \infty))$ are non-decreasing, $q_{2}>0, \omega_{1} \geqslant 0, q_{1} \in$ $C^{0}([0, b]), \omega_{2} \in C^{0}((0, \infty))$ are non-increasing, $q_{1} \geqslant 0, \omega_{2}>0, \omega_{1}+\omega_{2}$ is nonincreasing on $(0, \delta]$ with $\delta \in(0, T]$, and

$$
\int_{0}^{1} \omega_{2}\left(g^{-1}(s)\right) \mathrm{d} s<\infty
$$


Remark 1.1. Since $\omega_{2}\left(g^{-1}(t)\right)$ is non-increasing positive on $(0, \infty)$ by $\left(H_{1}\right)$ and $\left(H_{3}\right)$, the condition $\int_{0}^{1} \omega_{2}\left(g^{-1}(s)\right) \mathrm{d} s<\infty$ in $\left(H_{3}\right)$ implies $\int_{0}^{c} \omega_{2}\left(g^{-1}(s)\right) \mathrm{d} s<\infty$ for each $c \in(0, \infty)$.

We denote by $\|x\|=\max \{|x(t)|: t \in J\}$ and $\|x\|_{L_{1}}=\int_{0}^{T}|x(t)| \mathrm{d} t$ the norm in the Banach spaces $C^{0}(J)$ and $L_{1}(J)$, respectively.

To prove existence results for BVPs (1.1), (1.2) and (1.1), (1.3) we use regularization and sequential techniques. First, we define a family of auxiliary regular differential equations depending on $n \in \mathbb{N}$ and using the topological transversality theorem (see, for example, [1]) given in Lemma 2.1 we obtain a general existence principle (Lemma 2.2). Using this principle we show that the sequence of auxiliary BVPs has a sequence $\left\{x_{n}\right\}$ of positive solutions (Lemmas 2.3 and 2.4). Applying the Arzelà-Ascoli Theorem we can select a convergent subsequence of $\left\{x_{n}\right\}$ and then the Lebesgue-dominated convergence theorem shows that its limit is a solution of our BVP (Theorems 3.1 and 3.2). Example 3.3 demonstrates the application of our existence results.

\section{Auxiliary BVPs}

Let

$$
\mathbb{N}_{0}=\left\{n: n \in \mathbb{N}, n>\max \left\{\frac{2}{b}, \frac{1}{\alpha(0)}, \frac{1}{\varepsilon_{0}}, \frac{1}{\delta}\right\}\right\}
$$

where the positive numbers $b, \varepsilon_{0}, \delta$ and the function $\alpha$ are given by $\left(H_{2}\right)$ and $\left(H_{3}\right)$. For $n \in \mathbb{N}_{0}$, define $p_{n} \in C^{0}(\mathbb{R}), \tilde{f}_{n} \in \operatorname{Car}\left(J \times \mathbb{R}_{b} \times \mathbb{R}\right)$ and $f_{n} \in \operatorname{Car}\left(J \times \mathbb{R}^{2}\right)$ by

$$
\begin{gathered}
p_{n}(x)= \begin{cases}0 & \text { if } x>b, \\
n(b-x) & \text { if } b-(1 / n)<x \leqslant b, \\
1 & \text { if }(1 / n)<x \leqslant b-(1 / n), \\
n x & \text { if } 0<x \leqslant(1 / n) \\
0 & \text { if } x \leqslant 0,\end{cases} \\
\tilde{f}_{n}(t, x, y)= \begin{cases}f(t, x, \alpha(t)) & \text { for }(t, x, y) \in J \times \mathbb{R}_{b} \times[\alpha(t), \infty), \\
f(t, x, y) & \text { for }(t, x, y) \in J \times \mathbb{R}_{b} \times[(1 / n), \alpha(t)), \\
f(t, x,(1 / n)) & \text { for }(t, x, y) \in J \times \mathbb{R}_{b} \times(-\infty,(1 / n)),\end{cases} \\
f_{n}(t, x, y)= \begin{cases}p_{n}(x) \tilde{f}_{n}(t, x, y) & \text { for }(t, x, y) \in J \times \mathbb{R}_{b} \times \mathbb{R}, \\
0 & \text { for }(t, y) \in J \times \mathbb{R}, x=b .\end{cases}
\end{gathered}
$$

Then $\left(H_{2}\right)$ and $\left(H_{3}\right)$ give

$$
0 \leqslant f_{n}(t, x, y) \leqslant\left(q_{1}(x)+q_{2}(x)\right)\left(\omega_{1}(y)+\omega_{2}(y)\right)
$$

for a.e. $t \in J$ and each $(x, y) \in[0, b) \times(0, \alpha(t)]$.

Finally, define $g_{*} \in C^{0}(\mathbb{R})$ by

$$
g_{*}(u)= \begin{cases}g(u) & \text { for } u \in[0, \infty), \\ -g(-u) & \text { for } u \in(-\infty, 0) .\end{cases}
$$


Consider the family of regular differential equations

$$
\left(g_{*}\left(x^{\prime}(t)\right)\right)^{\prime}=\lambda \mu f_{n}\left(t, x(t), x^{\prime}(t)\right)
$$

depending on the parameters $n \in \mathbb{N}_{0}$ and $\lambda \in[0,1]$ together with the auxiliary boundary conditions

$$
x^{\prime}(0)=0, \quad x(T)=b-(1 / n) .
$$

For the solvability of BVPs $(\mathrm{R})_{n}^{1},(\mathrm{~B})_{n}$ and $(\mathrm{R})_{n}^{1},(1.3)$ we use the existence principle, whose proof is based on a well-known result in the literature (Lemma 2.1).

Lemma 2.1 (see [1]). Let $\Omega$ be a relatively open set of a convex set $\Phi$ in a Banach space $E$ and $p \in \Omega$. If

(a) $\mathcal{K}:[0,1] \times \bar{\Omega} \rightarrow \Phi$ is a compact operator,

(b) $\mathcal{K}(0, x)=p$ for $x \in \bar{\Omega}$, and

(c) $\mathcal{K}(\lambda, x) \neq x$ for $\lambda \in(0,1)$ and $x \in \partial \Omega$,

then $\mathcal{K}(1, \cdot)$ has a fixed point in $\bar{\Omega}$.

Lemma 2.2. Let $F \in \operatorname{Car}\left(J \times \mathbb{R}^{2}\right), g_{*}$ be given by (2.2), $d \in \mathbb{R}$ and let there exist positive constants $P_{0}, P_{1}$ independent of $\lambda, P_{0}>|d|$, such that

$$
\|x\| \neq P_{0}, \quad\left\|x^{\prime}\right\| \neq P_{1}
$$

for any solution $x$ to BVP

$$
\begin{gathered}
\left(g_{*}\left(x^{\prime}(t)\right)\right)^{\prime}=\lambda F\left(t, x(t), x^{\prime}(t)\right), \\
x^{\prime}(0)=0, \quad x(T)=d
\end{gathered}
$$

with $\lambda \in(0,1)$. Then there exists a solution of $B V P(2.3)^{1},(2.4)$.

Proof. Set

$$
\Omega=\left\{x: x \in C^{1}(J),\|x\|<P_{0},\left\|x^{\prime}\right\|<P_{1}\right\}
$$

and let $\mathcal{K}:[0,1] \times \bar{\Omega} \rightarrow C^{1}(J)$,

$$
\mathcal{K}(\lambda, x)(t)=d-\int_{t}^{T} g_{*}^{-1}\left(\lambda \int_{0}^{s} F\left(v, x(v), x^{\prime}(v)\right) \mathrm{d} v\right) \mathrm{d} s .
$$

Then $x$ is a fixed point of the operator $\mathcal{K}(\lambda, \cdot)$ if and only if $x$ is a solution of BVP $(2.3)^{\lambda},(2.4)$. To prove the solvability of BVP $(2.3)^{1},(2.4)$ we use Lemma 2.1 (with $E=\Phi=C^{1}(J)$ and $\left.p=d\right)$.

First we see that $\mathcal{K}(0, x)=d$ for $x \in \bar{\Omega}$. If $\mathcal{K}\left(\lambda_{0}, x_{0}\right)=x_{0}$ for some $\left(\lambda_{0}, x_{0}\right) \in(0,1) \times$ $\partial \Omega$, then $x_{0}$ is a solution of $\operatorname{BVP}(2.3)^{\lambda_{0}},(2.4)$ and $\left\|x_{0}\right\| \neq P_{0},\left\|x_{0}^{\prime}\right\| \neq P_{1}$ by our assumption, that is $x_{0} \notin \partial \Omega$, contrary to $x_{0} \in \partial \Omega$. 
It remains to show that $\mathcal{K}$ is a compact operator. For this let $\left\{\left(\lambda_{n}, x_{n}\right)\right\} \subset[0,1] \times \bar{\Omega}$ be a convergent sequence, $\lim _{n \rightarrow \infty} \lambda_{n}=\lambda_{0}, \lim _{n \rightarrow \infty} x_{n}=x_{0}$. Then there is a positive constant $L$ such that $\left\|x_{n}\right\| \leqslant L,\left\|x_{n}^{\prime}\right\| \leqslant L$ for $n \in \mathbb{N}$, and since $F \in \operatorname{Car}\left(J \times \mathbb{R}^{2}\right)$,

$$
\left|F\left(t, x_{n}(t), x_{n}^{\prime}(t)\right)\right| \leqslant \gamma(t) \text { for a.e. } t \in J \text { and each } n \in \mathbb{N},
$$

where $\gamma \in L_{1}(J)$. Hence

$$
\lim _{n \rightarrow \infty} \lambda_{n} \int_{0}^{t} F\left(s, x_{n}(s), x_{n}^{\prime}(s)\right) \mathrm{d} s=\lambda_{0} \int_{0}^{t} F\left(s, x(s), x^{\prime}(s)\right) \mathrm{d} s
$$

uniformly on $J$ by the Lebesgue-dominated convergence theorem, and so the sequence

$$
\left\{g_{*}\left(\left(\mathcal{K}\left(\lambda_{n}, x_{n}\right)\right)^{\prime}(t)\right)\right\}=\left\{\lambda_{n} \int_{0}^{t} F\left(s, x_{n}(s), x_{n}^{\prime}(s)\right) \mathrm{d} s\right\}
$$

is uniformly convergent on $J$ to $\lambda_{0} \int_{0}^{t} F\left(s, x_{0}(s), x_{0}^{\prime}(s)\right) \mathrm{d} s$. Now from the equalities (for $n \in \mathbb{N}$ and $t \in J)$

$$
\left|\left(\mathcal{K}\left(\lambda_{n}, x_{n}\right)\right)^{\prime}(t)-\left(\mathcal{K}\left(\lambda_{0}, x_{0}\right)\right)^{\prime}(t)\right|=\left|g_{*}^{-1}\left[g_{*}\left(\left(\mathcal{K}\left(\lambda_{n}, x_{n}\right)\right)^{\prime}(t)\right)\right]-g_{*}^{-1}\left[g_{*}\left(\left(\mathcal{K}\left(\lambda_{0}, x_{0}\right)\right)^{\prime}(t)\right)\right]\right|
$$

and $g_{*}$ being continuous increasing on $\mathbb{R}$, we deduce that

$$
\lim _{n \rightarrow \infty}\left(\mathcal{K}\left(\lambda_{n}, x_{n}\right)\right)^{\prime}(t)=\left(\mathcal{K}\left(\lambda_{0}, x_{0}\right)\right)^{\prime}(t) \quad \text { uniformly on } J .
$$

Thus $\lim _{n \rightarrow \infty} \mathcal{K}\left(\lambda_{n}, x_{n}\right)=\mathcal{K}\left(\lambda_{0}, x_{0}\right)$ in $C^{1}(J)$ since $\mathcal{K}\left(\lambda_{n}, x_{n}\right)(T)=\mathcal{K}\left(\lambda_{0}, x_{0}\right)(T)=d$ for $n \in \mathbb{N}$. We have proved that $\mathcal{K}$ is a continuous operator. To prove the relative compactness of $\mathcal{K}([0,1] \times \bar{\Omega})$ in $C^{1}(J)$, let $\left\{\left(\lambda_{m}, x_{m}\right)\right\} \subset[0,1] \times \bar{\Omega}$. Then (2.5) (with $m$ instead of $n$ ) is satisfied with a $\gamma \in L_{1}(J)$ and we can verify that

$$
\left|g_{*}\left(\left(\mathcal{K}\left(\lambda_{m}, x_{m}\right)\right)^{\prime}(t)\right)\right| \leqslant\|\gamma\|_{L_{1}}, \quad\left|\mathcal{K}\left(\lambda_{m}, x_{m}\right)(t)\right| \leqslant|d|+T g_{*}^{-1}\left(\|\gamma\|_{L_{1}}\right)
$$

and

$$
\left|g_{*}\left(\left(\mathcal{K}\left(\lambda_{m}, x_{m}\right)\right)^{\prime}\left(t_{2}\right)\right)-g_{*}\left(\left(\mathcal{K}\left(\lambda_{m}, x_{m}\right)\right)^{\prime}\left(t_{1}\right)\right)\right| \leqslant\left|\int_{t_{1}}^{t_{2}} \gamma(t) \mathrm{d} t\right|
$$

for $t, t_{1}, t_{2} \in J$ and $m \in \mathbb{N}$. Therefore, $\left\{\mathcal{K}\left(\lambda_{m}, x_{m}\right)\right\}$ is bounded in $C^{1}(J)$ and from (2.6) and $g_{*}$ being increasing on $\mathbb{R}$ it follows that $\left\{\left(\mathcal{K}\left(\lambda_{m}, x_{m}\right)\right)^{\prime}(t)\right\}$ is equicontinuous on $J$. Consequently, there is a subsequence of $\left\{\mathcal{K}\left(\lambda_{m}, x_{m}\right)\right\}$ converging in $C^{1}(J)$ by the Arzelà-Ascoli Theorem. This completes the proof.

Let

$$
Q(u)=\int_{0}^{u}\left(q_{1}(s)+q_{2}(s)\right) \mathrm{d} s, \quad u \in[0, b),
$$

and

$$
H(u)=\int_{0}^{g(u)} \frac{g^{-1}(s)}{\omega_{1}\left(g^{-1}(s)\right)+\omega_{2}\left(g^{-1}(s)\right)} \mathrm{d} s, \quad u \in[0, \infty) .
$$


Then, by $\left(H_{3}\right), Q \in C^{0}([0, b))$ and $H \in C^{0}([0, \infty))$ are increasing. In addition, if

$$
\int_{0}^{b} q_{2}(t) \mathrm{d} t<\infty
$$

then $Q \in C^{0}([0, b])$.

Lemma 2.3. Let (2.7) be satisfied and let there exist $\mu_{0}>0$ such that

$$
\int_{0}^{b} \frac{1}{H^{-1}\left(\mu_{0} Q(s)\right)} \mathrm{d} s<\infty .
$$

Then the function

$$
r(\mu)=\int_{0}^{b} \frac{1}{H^{-1}(\mu Q(s))} \mathrm{d} s
$$

is continuous and decreasing on $\left(0, \mu_{0}\right]$.

Proof. We first see that (2.8) shows that the interval $\left[0, \mu_{0} Q(b)\right)$ belongs to the domain of $H^{-1}$. By $\left(H_{3}\right)$, there are positive constants $k_{1}, k_{2}, k_{1}<k_{2}$, and $b_{0} \in(0, b)$ such that $k_{1} \leqslant q_{1}(u)+q_{2}(u) \leqslant k_{2}$ for $u \in\left[0, b_{0}\right]$, and so

$$
k_{1} u \leqslant Q(u) \leqslant k_{2} u \text { for } u \in\left[0, b_{0}\right] .
$$

Then $H^{-1}\left(\mu_{0} Q(u)\right) \leqslant H^{-1}\left(\mu_{0} k_{2} u\right)$ for $u \in\left[0, b_{1}\right]$, where $0<b_{1}<\min \left\{b_{0}, Q(b) / k_{2}\right\}$ and (see $(2.8))$

$$
\int_{0}^{b_{1}} \frac{1}{H^{-1}\left(\mu_{0} k_{2} s\right)} \mathrm{d} s \leqslant \int_{0}^{b_{1}} \frac{1}{H^{-1}\left(\mu_{0} Q(s)\right)} \mathrm{d} s<\int_{0}^{b} \frac{1}{H^{-1}\left(\mu_{0} Q(s)\right)} \mathrm{d} s<\infty,
$$

which shows that

$$
\int_{0}^{\mu_{0} k_{2} b_{1}} \frac{1}{H^{-1}(s)} \mathrm{d} s<\infty .
$$

Let $\mu \in\left(0, \mu_{0}\right)$. Now from the inequalities (see $(2.10)$ )

$$
\begin{array}{ll}
H^{-1}(\mu Q(u)) \geqslant H^{-1}\left(\mu k_{1} u\right) & \text { for } u \in\left[0, b_{0}\right], \\
H^{-1}(\mu Q(u)) \geqslant H^{-1}\left(\mu Q\left(b_{0}\right)\right) & \text { for } u \in\left[b_{0}, b\right],
\end{array}
$$

we have

$$
\int_{0}^{b} \frac{1}{H^{-1}(\mu Q(s))} \mathrm{d} s \leqslant \int_{0}^{b_{0}} \frac{1}{H^{-1}\left(\mu k_{1} s\right)} \mathrm{d} s+\frac{b-b_{0}}{H^{-1}\left(\mu Q\left(b_{0}\right)\right)},
$$

and then using (2.11) we deduce that the function $r$ given by $(2.9)$ is defined on $\left(0, \mu_{0}\right]$. Since the function $v(u, \mu)=H^{-1}(\mu Q(u))$ is continuous on $[0, b] \times\left[0, \mu_{0}\right]$ and $v(u, \cdot)$ is increasing on $\left[0, \mu_{0}\right]$ for each $u \in(0, b]$, it follows that $r$ is continuous and decreasing on $\left(0, \mu_{0}\right]$. 
Lemma 2.4. Let (2.7) be satisfied and let $\mu_{*}$ be a positive number such that

$$
\int_{0}^{b} \frac{1}{H^{-1}\left(\mu_{*} Q(s)\right)} \mathrm{d} s=T .
$$

Then for each $\mu \in\left(0, \mu_{*}\right)$ there exists $n_{\mu} \in \mathbb{N}$ such that $B V P(\mathrm{R})_{n}^{1},(\mathrm{~B})_{n}$ with $n \in \mathbb{N}_{\mu}=$ $\left\{n: n \in \mathbb{N}_{0}, n \geqslant n_{\mu}\right\}$ has a solution $x_{n}$ and

$$
\frac{1}{n_{\mu}} \leqslant x_{n}(t) \leqslant b-\frac{1}{n}, \quad 0 \leqslant x_{n}^{\prime}(t) \leqslant \alpha(t) \quad \text { for } t \in J
$$

Proof. Fix $\mu \in\left(0, \mu_{*}\right)$. By Lemma 2.3, there exist $n_{\mu} \in \mathbb{N}$ such that

$$
\int_{1 / n_{\mu}}^{b-1 / n_{\mu}} \frac{1}{H^{-1}(\mu Q(s))} \mathrm{d} s \geqslant T .
$$

Fix $n \in \mathbb{N}_{\mu}$. Consider the family of BVPs $(\mathrm{R})_{n}^{\lambda},(\mathrm{B})_{n}$ for $0<\lambda<1$. First, we show that any solution $x$ to $(\mathrm{R})_{n}^{\lambda},(\mathrm{B})_{n}$ satisfies

$$
x(t) \geqslant 0 \quad \text { for } t \in J .
$$

To see this suppose that $\min \{x(t): t \in J\}=x\left(t_{0}\right)<0$. Then $t_{0} \in[0, T), x\left(t_{1}\right)=0$ for a $t_{1} \in\left(t_{0}, T\right)$ and $x<0$ on $\left[t_{0}, t_{1}\right)$ since $x(T)=b-1 / n>0$ and also $x^{\prime}\left(t_{0}\right)=0$, which is clear for $t_{0} \in(0, T)$ and it follows from $(\mathrm{B})_{n}$ for $t_{0}=0$. Moreover,

$$
\left(g_{*}\left(x^{\prime}(t)\right)\right)^{\prime}=\lambda \mu f_{n}\left(t, x(t), x^{\prime}(t)\right)=0 \quad \text { for a.e. } t \in\left[t_{0}, t_{1}\right],
$$

and so $x(t)=x\left(t_{0}\right)<0$ for $t \in\left[t_{0}, t_{1}\right)$, which yields $x\left(t_{1}\right)=x\left(t_{0}\right)<0$, contrary to $x\left(t_{1}\right)=0$. Now, from $\left(g_{*}\left(x^{\prime}(t)\right)\right)^{\prime}=\lambda \mu f_{n}\left(t, x(t), x^{\prime}(t)\right) \geqslant 0$ for a.e. $t \in J$ we have $x^{\prime} \geqslant 0$ on $J$, and consequently $(2.15)$ is true and $x(t) \leqslant x(T)=b-(1 / n)$ for $t \in J$. Next we will show that

$$
(0 \leqslant) x^{\prime}(t) \leqslant \alpha(t) \text { for } t \in J
$$

If not, $x^{\prime}\left(t_{1}\right)>\alpha\left(t_{1}\right)$ for some $t_{1} \in(0, T)$ and then from $x^{\prime}(0)=0$ and $\alpha(0)>0$ we deduce that $x^{\prime}\left(t_{*}\right)=\alpha\left(t_{*}\right)$ and $x^{\prime}>\alpha$ on $\left(t_{*}, t_{1}\right]$ with a $t_{*} \in\left(0, t_{1}\right)$. Therefore,

$$
\left(g_{*}\left(x^{\prime}(t)\right)\right)^{\prime}=\lambda \mu f_{n}(t, x(t), \alpha(t))=0 \quad \text { for a.e. } t \in\left[t_{*}, t_{1}\right],
$$

hence $g_{*}\left(x^{\prime}(t)\right)=g\left(\alpha\left(t_{*}\right)\right)$ for $t \in\left[t_{*}, t_{1}\right]$, and so $\alpha\left(t_{*}\right)=x^{\prime}\left(t_{*}\right)=x^{\prime}\left(t_{1}\right)>\alpha\left(t_{1}\right)$, which contradicts $\alpha$ being non-decreasing on $J$ by $\left(H_{2}\right)$. Now $(\mathrm{B})_{n},(2.15),(2.16)$ together with Lemma 2.2 (with $F(t, x, y)=\mu f_{n}(t, x, y), P_{0}=b$ and $\left.P_{1}=\|\alpha\|+1\right)$ imply that $(\mathrm{R})_{n}^{1}$, $(\mathrm{B})_{n}$ has a solution $x_{n}$. In addition (argue as above)

$$
0 \leqslant x_{n}(t) \leqslant b-(1 / n), \quad 0 \leqslant x_{n}^{\prime}(t) \leqslant \alpha(t) \text { for } t \in J .
$$

To prove (2.13) it remains to verify that $x_{n} \geqslant 1 / n_{\mu}$ on $J$. By $(2.1)$,

$$
\left(g_{*}\left(x_{n}^{\prime}(t)\right)\right)^{\prime}=\left(g\left(x_{n}^{\prime}(t)\right)\right)^{\prime} \leqslant \mu\left[q_{1}\left(x_{n}(t)\right)+q_{2}\left(x_{n}(t)\right)\right]\left[\omega\left(x_{n}^{\prime}(t)\right)+\omega_{2}\left(x_{n}^{\prime}(t)\right)\right]
$$


for a.e. $t \in J$. Then, integrating the inequality

$$
\frac{\left(g\left(x_{n}^{\prime}(t)\right)\right)^{\prime} x_{n}^{\prime}(t)}{\omega_{1}\left(x_{n}^{\prime}(t)\right)+\omega_{2}\left(x_{n}^{\prime}(t)\right)} \leqslant \mu\left[q_{1}\left(x_{n}(t)\right)+q_{2}\left(x_{n}(t)\right)\right] x_{n}^{\prime}(t)
$$

from 0 to $t \in(0, T]$, we get

$$
\begin{aligned}
\int_{0}^{g\left(x_{n}^{\prime}(t)\right)} \frac{g^{-1}(s)}{\omega_{1}\left(g^{-1}(s)\right)+\omega_{2}\left(g^{-1}(s)\right)} \mathrm{d} s & \leqslant \mu \int_{x_{n}(0)}^{x_{n}(t)}\left(q_{1}(s)+q_{2}(s)\right) \mathrm{d} s \\
& \leqslant \mu \int_{0}^{x_{n}(t)}\left(q_{1}(s)+q_{2}(s)\right) \mathrm{d} s
\end{aligned}
$$

for $t \in J$. Hence $H\left(x_{n}^{\prime}(t)\right) \leqslant \mu Q\left(x_{n}(t)\right)$ for $t \in J$ and integrating from $\bar{t}=\min \{t: t \in$ $\left.J, x_{n}(t)>0\right\}$ to $T$ the inequality

$$
\frac{x_{n}^{\prime}(t)}{H^{-1}\left(\mu Q\left(x_{n}(t)\right)\right)} \leqslant 1 \quad \text { for } t \in(\bar{t}, T],
$$

we have

$$
\int_{x_{n}(\bar{t})}^{b-1 / n} \frac{1}{H^{-1}(\mu Q(s))} \mathrm{d} s \leqslant T .
$$

Since $1 / n \leqslant 1 / n_{\mu},(2.14)$ shows that $x_{n}(\bar{t}) \geqslant 1 / n_{\mu}$, so $\bar{t}=0$ and $x_{n}(0) \geqslant 1 / n_{\mu}$. We have proved the validity of $(2.13)$.

Lemma 2.5. Let $0<c<b$ and let $\mu_{0}$ be a positive number such that

$$
\int_{0}^{c} \frac{1}{H^{-1}\left(\mu_{0} Q(s)\right)} \mathrm{d} s=T .
$$

Then for each $\mu \in\left(0, \mu_{0}\right)$ there exists $n_{\mu} \in \mathbb{N}$ such that $B V P(\mathrm{R})_{n}^{1}$, (1.3) with $n \in \mathbb{N}_{\mu}=$ $\left\{n: n \in \mathbb{N}_{0}, n \geqslant n_{\mu}\right\}$ has a solution $x_{n}$ and

$$
\frac{1}{n_{\mu}} \leqslant x_{n}(t) \leqslant c \leqslant b-\frac{1}{n}, \quad 0 \leqslant x_{n}^{\prime}(t) \leqslant \alpha(t) \quad \text { for } t \in J .
$$

Proof. Arguing as in the proof of Lemma 2.3 we can prove that the function

$$
r_{*}(\mu)=\int_{0}^{c}\left[1 / H^{-1}(\mu Q(s))\right] \mathrm{d} s
$$

is continuous decreasing on $\left(0, \mu_{0}\right]$. Fix $\mu \in\left(0, \mu_{0}\right)$. Then there exists $n_{\mu} \in \mathbb{N}, n_{\mu} \geqslant$ $1 /(b-c)$ such that

$$
\int_{1 / n_{\mu}}^{c} \frac{1}{H^{-1}(\mu Q(s))} \mathrm{d} s \geqslant T .
$$

Let $n \in \mathbb{N}_{\mu}$. Consider the family of BVPs $(\mathrm{R})_{n}^{\lambda}$, (1.3) for $0<\lambda<1$. An analysis similar to that in the proof of Lemma 2.4 shows that any solution $x$ to $(\mathrm{R})_{n}^{\lambda},(1.3)$ satisfies the inequality

$$
0 \leqslant x(t) \leqslant c, \quad 0 \leqslant x^{\prime}(t) \leqslant \alpha(t), \quad t \in J
$$


and so Lemma 2.2 (with $P_{0}=c+1$ and $P_{1}=\|\alpha\|+1$ ) guarantees that there exists a solution $x_{n}$ of $\operatorname{BVP}(\mathrm{R})_{n}^{1},(1.3)$ for $n \in \mathbb{N}_{\mu}$. Then

$$
0 \leqslant x_{n}(t) \leqslant c \leqslant b-(1 / n), \quad 0 \leqslant x_{n}^{\prime}(t) \leqslant \alpha(t), \quad t \in J,
$$

and integrating (2.18) from $\bar{t}=\min \left\{t: t \in J, x_{n}(t)>0\right\}$ to $T$, we get

$$
\int_{x_{n}(\bar{t})}^{c} \frac{1}{H^{-1}(\mu Q(s))} \mathrm{d} s \leqslant T .
$$

Now $(2.21)$ shows that $x_{n}(\bar{t}) \geqslant 1 / n_{\mu}$, so $\bar{t}=0$ and $x_{n}(0) \geqslant 1 / n_{\mu}$, which completes the proof of (2.20).

\section{Existence results and an example}

Theorem 3.1. Let assumptions $\left(H_{1}\right)-\left(H_{3}\right),(2.7)$ be satisfied and let (2.12) be true with a positive number $\mu_{*}$. Then $B V P(1.1),(1.2)$ has a solution $x$ for each $\mu \in\left(0, \mu_{*}\right)$ and

$$
0<x(t) \leqslant b, \quad 0 \leqslant x^{\prime}(t) \leqslant \alpha(t) \quad \text { for } t \in J .
$$

Proof. Fix $\mu \in\left(0, \mu_{*}\right)$. By Lemma 2.4, there exists $n_{\mu} \in \mathbb{N}$ such that BVP $(\mathrm{R})_{n}^{1},(\mathrm{~B})_{n}$ has a solution $x_{n}$ satisfying (2.13) for each $n \in \mathbb{N}_{\mu}$ with $\mathbb{N}_{\mu}$ given in Lemma 2.4. Then (see (2.1) and (2.13))

$$
\left(g\left(x_{n}^{\prime}(t)\right)\right)^{\prime} \leqslant \mu\left[q_{1}\left(x_{n}(t)\right)+q_{2}\left(x_{n}(t)\right)\right]\left[\omega_{1}\left(x_{n}^{\prime}(t)\right)+\omega_{2}\left(x_{n}^{\prime}(t)\right)\right]
$$

for a.e $t \in J$ and each $n \in \mathbb{N}_{\mu}$. Arguing as in the proof of Lemma 2.4 we can see that (see $(2.17))$

$$
H\left(x_{n}^{\prime}(t)\right) \leqslant \mu\left[Q\left(x_{n}(t)\right)-Q\left(x_{n}(0)\right)\right] \quad \text { for } t \in J, n \in \mathbb{N}_{\mu} .
$$

Now let $\varepsilon, \varepsilon_{0}$ and $\nu$ be given by $\left(H_{2}\right)$. If there exists a subsequence $\left\{x_{k_{n}}\right\}$ of $\left\{x_{n}\right\}_{n \in \mathbb{N}_{\mu}}$ such that $x_{k_{n}}^{\prime}\left(\tau_{n}\right)=\varepsilon_{0}$ with $\lim _{n \rightarrow \infty} \tau_{n}=0$, then letting $n \rightarrow \infty$ in (see (3.3))

$$
H\left(x_{k_{n}}^{\prime}\left(\tau_{n}\right)\right) \leqslant \mu\left[Q\left(x_{k_{n}}\left(\tau_{n}\right)\right)-Q\left(x_{k_{n}}(0)\right)\right],
$$

we get $H\left(\varepsilon_{0}\right) \leqslant 0$ since $0 \leqslant x_{k_{n}}\left(\tau_{n}\right)-x_{k_{n}}(0) \leqslant\|\alpha\| \tau_{n}$, contrary to $H\left(\varepsilon_{0}\right)>0$. Consequently, there is a $\nu_{0} \in(0, \nu]$ such that $x_{n}^{\prime}(t) \leqslant \varepsilon_{0}$ for $t \in\left[0, \nu_{0}\right]$ and $n \in \mathbb{N}_{\mu}$, and then $\left(H_{2}\right)$ and (2.13) imply that $f_{n}\left(t, x_{n}(t), x_{n}^{\prime}(t)\right) \geqslant \varepsilon$ for a.e. $t \in\left[0, \nu_{0}\right]$ and each $n \in \mathbb{N}_{\mu}$. Hence $g\left(x_{n}^{\prime}(t)\right) \geqslant \varepsilon t$ for $t \in\left[0, \nu_{0}\right]$ and since $x_{n}^{\prime}$ is non-decreasing on $J$, we have

$$
x_{n}^{\prime}(t) \geqslant \chi(t) \quad \text { for } t \in J \text { and } n \in \mathbb{N}_{\mu},
$$

with

$$
\chi(t)= \begin{cases}g^{-1}(\varepsilon t) & \text { for } t \in\left[0, \nu_{0}\right] \\ g^{-1}\left(\varepsilon \nu_{0}\right) & \text { for } t \in\left(\nu_{0}, T\right]\end{cases}
$$

Then

$$
\omega_{2}\left(x_{n}^{\prime}(t)\right) \leqslant \omega_{2}(\chi(t)) \quad \text { for } t \in(0, T], n \in \mathbb{N}_{\mu} .
$$


Using the assumption (see $\left(H_{3}\right)$ and Remark 1.1) $\int_{0}^{1} \omega_{2}\left(g^{-1}(s)\right) \mathrm{d} s<\infty$ we see that $\omega_{2}(\chi(t)) \in L_{1}(J)$. In addition, from $(\mathrm{B})_{n}$ and (3.4) we have

$$
x_{n}(t)=x_{n}(b)-\int_{t}^{b} x_{n}^{\prime}(s) \mathrm{d} s<b-\int_{t}^{b} \chi(s) \mathrm{d} s,
$$

and so

$$
x_{n}(t)<\eta(t) \quad \text { for } t \in J, n \in \mathbb{N}_{\mu},
$$

where

$$
\eta(t)= \begin{cases}b-g^{-1}\left(\varepsilon \nu_{0}\right)\left(b-\nu_{0}\right) & \text { for } t \in\left[0, \nu_{0}\right] \\ b-g^{-1}\left(\varepsilon \nu_{0}\right)(b-t) & \text { for } t \in\left(\nu_{0}, T\right]\end{cases}
$$

Hence

$$
q_{2}\left(x_{n}(t)\right) \leqslant q_{2}(\eta(t)) \quad \text { for } t \in J, n \in \mathbb{N}_{\mu}
$$

and $q_{2}(\eta(t)) \in L_{1}(J)$, which follows from assumption (2.7) and the inequalities

$$
\begin{aligned}
\int_{0}^{b} q_{2}(\eta(t)) \mathrm{d} t & =\nu_{0} q_{2}\left(b-g^{-1}\left(\varepsilon \nu_{0}\right)\left(b-\nu_{0}\right)\right)+\int_{\nu_{0}}^{b} q_{2}\left(b-g^{-1}\left(\varepsilon \nu_{0}\right)(b-t)\right) \mathrm{d} t \\
& =\nu_{0} q_{2}\left(b-g^{-1}\left(\varepsilon \nu_{0}\right)\left(b-\nu_{0}\right)\right)+\frac{1}{g^{-1}\left(\varepsilon \nu_{0}\right)} \int_{b-g^{-1}\left(\varepsilon \nu_{0}\right)\left(b-\nu_{0}\right)}^{b} q_{2}(t) \mathrm{d} t \\
& \leqslant \nu_{0} q_{2}\left(b-g^{-1}\left(\varepsilon \nu_{0}\right)\left(b-\nu_{0}\right)\right)+\frac{1}{g^{-1}\left(\varepsilon \nu_{0}\right)} \int_{0}^{b} q_{2}(t) \mathrm{d} t .
\end{aligned}
$$

Now, from (2.13), (3.2), (3.5) and (3.6) we have

$$
(0 \leqslant)\left(g\left(x_{n}^{\prime}(t)\right)\right)^{\prime} \leqslant \mu\left[q_{1}(0)+q_{2}(\eta(t))\right]\left[\omega_{1}(\alpha(t))+\omega_{2}(\chi(t))\right]
$$

for a.e. $t \in J$ and each $n \in \mathbb{N}_{\mu}$. Hence

$$
0 \leqslant g\left(x_{n}^{\prime}\left(t_{2}\right)\right)-g\left(x_{n}^{\prime}\left(t_{1}\right)\right) \leqslant \mu \int_{t_{1}}^{t_{2}}\left[q_{1}(0)+q_{2}(\eta(t))\right]\left[\omega_{1}(\alpha(t))+\omega_{2}(\chi(t))\right] \mathrm{d} t
$$

for $0 \leqslant t_{1} \leqslant t_{2} \leqslant T$ and $n \in \mathbb{N}_{\mu}$, and since $\left[q_{1}(0)+q_{2}(\eta(t))\right]\left[\omega_{1}(\alpha(t))+\omega_{2}(\chi(t))\right] \in L_{1}(J)$, $\left\{g\left(x_{n}^{\prime}(t)\right)\right\}_{n \in \mathbb{N}_{\mu}}$ is equicontinuous on $J$ and then $\left\{x_{n}^{\prime}(t)\right\}_{n \in \mathbb{N}_{\mu}}$ is equicontinuous on $J$ too since $g$ is continuous increasing on $[0, \infty)$ by $\left(H_{1}\right)$. Applying the Arzelà-Ascoli Theorem, going if necessary to a subsequence, we can assume that $\left\{x_{n}^{\prime}\right\}_{n \in \mathbb{N}_{\mu}}$ is convergent in $C^{1}(J)$ and $\operatorname{let}_{\lim _{n \rightarrow \infty}} x_{n}=x$. Then $x \in C^{1}(J), 1 / n_{\mu} \leqslant x(t) \leqslant \eta(t), \chi(t) \leqslant x^{\prime}(t) \leqslant \alpha(t)$ for $t \in J, x^{\prime}(0)=0$ and $x(T)=b$. As

$$
\lim _{n \rightarrow \infty} f_{n}\left(t, x_{n}(t), x_{n}^{\prime}(t)\right)=f\left(t, x(t), x^{\prime}(t)\right) \quad \text { for a.e. } t \in J
$$

and

$$
0 \leqslant f_{n}\left(t, x_{n}(t), x_{n}^{\prime}(t)\right) \leqslant \mu\left[q_{1}(0)+q_{2}(\eta(t))\right]\left[\omega_{1}(\alpha(t))+\omega_{2}(\chi(t))\right]\left(\in L_{1}(J)\right)
$$


for a.e. $t \in J$ and each $n \in \mathbb{N}_{\mu}$, taking the limit as $n \rightarrow \infty$ in

$$
g\left(x_{n}^{\prime}(t)\right)=\mu \int_{0}^{t} f_{n}\left(s, x_{n}(s), x_{n}^{\prime}(s)\right) \mathrm{d} s, \quad t \in J, n \in \mathbb{N}_{\mu},
$$

we get

$$
g\left(x^{\prime}(t)\right)=\mu \int_{0}^{t} f\left(s, x(s), x^{\prime}(s)\right) \mathrm{d} s, \quad t \in J
$$

by the Lebesgue-dominated convergence theorem. Therefore, $g\left(x^{\prime}\right) \in \mathrm{AC}(J)$ and $x$ is a solution of BVP (1.1), (1.2).

Theorem 3.2. Let assumptions $\left(H_{1}\right)-\left(H_{3}\right)$ be satisfied, $0<c<b$ and let (2.19) be true with a positive number $\mu_{0}$. Then $B V P(1.1),(1.3)$ has a solution $x$ for each $\mu \in\left(0, \mu_{0}\right)$ and

$$
0<x(t) \leqslant c, \quad 0 \leqslant x^{\prime}(t) \leqslant \alpha(t) \quad \text { for } t \in J .
$$

Proof. Fix $\mu \in\left(0, \mu_{0}\right)$. By Lemma 2.5, there exists $n_{\mu} \in \mathbb{N}$ such that BVP $(\mathrm{R})_{n}^{1},(1.3)$ has a solution $x_{n}$ satisfying (2.20) for each $n \in \mathbb{N}_{\mu}$ with $\mathbb{N}_{\mu}$ given in Lemma 2.5. Then (see (2.1) and (2.20))

$$
\left(g\left(x_{n}^{\prime}(t)\right)\right)^{\prime} \leqslant \mu\left[q_{1}(0)+q_{2}(c)\right]\left[\omega_{1}(\alpha(t))+\omega_{2}\left(x_{n}^{\prime}(t)\right)\right]
$$

for a.e. $t \in J$ and each $n \in \mathbb{N}_{\mu}$. We can now proceed analogously to the proof of Theorem 3.1 to show that

$$
\left(g\left(x_{n}^{\prime}(t)\right)\right)^{\prime} \leqslant \mu\left[q_{1}(0)+q_{2}(c)\right]\left[\omega_{1}(\alpha(t))+\omega_{2}(\chi(t))\right]\left(\in L_{1}(J)\right)
$$

for a.e. $t \in J$ and each $n \in \mathbb{N}_{\mu}$, and (the details are left to the reader) we can also show that there is a convergent in $\left(C^{1}(J)\right)$ subsequence of $\left\{x_{n}\right\}_{n \in \mathbb{N}_{\mu}}$ and its limit is a solution of BVP (1.1), (1.3).

It is easy to construct examples so that Theorems 3.1 and 3.2 can be applied in practice. To illustrate this we consider the following two examples.

Example 3.3. Let $b, r, \gamma$ and $\eta$ be positive constants, $\gamma \neq 1, \eta<r$. Consider the differential equation

$$
\left(\left|x^{\prime}(t)\right|^{r}\right)^{\prime}=\mu \frac{\operatorname{sgn}(b-x(t))}{|x(t)-b|^{\gamma}}\left(\frac{\operatorname{sgn} x^{\prime}(t)}{\left|x^{\prime}(t)\right|^{\eta}}-1\right)
$$

which is the special case of (1.1) with

$$
g(u)=|u|^{r}, \quad f(t, x, y)=\frac{\operatorname{sgn}(b-x)}{|x-b|^{\gamma}}\left(\frac{\operatorname{sgn} y}{|y|^{\eta}}-1\right) .
$$

Then $g$ satisfies assumption $\left(H_{1}\right)$. Assumption $\left(H_{2}\right)$ is satisfied with $\alpha(t)=1, \nu=T$ and for instance $\varepsilon=1 / b^{\gamma}, \varepsilon_{0}=1 / \sqrt[\eta]{2}$ and assumption $\left(H_{3}\right)$ is satisfied with $q_{1}(u)=0$, $q_{2}(u)=1 /(b-u)^{\gamma}, \omega_{1}(u)=0$ and $\omega_{2}(u)=1 / u^{\eta}$. Since $\eta<r$, we see that

$$
\int_{0}^{1} \omega_{2}\left(g^{-1}(s)\right) \mathrm{d} s=\int_{0}^{1}\left(1 / u^{\eta / r}\right) \mathrm{d} u<\infty
$$


Then

$$
\begin{aligned}
Q(u) & =\int_{0}^{u}\left(q_{1}(s)+q_{2}(s)\right) \mathrm{d} s \\
& =\int_{0}^{u} \frac{1}{(b-s)^{\gamma}} \mathrm{d} s \\
& =\frac{b^{1-\gamma}-(b-u)^{1-\gamma}}{1-\gamma}
\end{aligned}
$$

and

$$
\begin{aligned}
H(u) & =\int_{0}^{g(u)} \frac{g^{-1}(s)}{\omega_{1}\left(g^{-1}(s)\right)+\omega_{2}\left(g^{-1}(s)\right)} \mathrm{d} s \\
& =\int_{0}^{u^{r}} s^{(\eta+1) / r} \mathrm{~d} s \\
& =\frac{r}{\eta+r+1} u^{\eta+r+1} .
\end{aligned}
$$

Hence

$$
H^{-1}(u)=\sqrt[\eta+r+1]{\frac{\eta+r+1}{r} u}
$$

and

$$
H^{-1}(\mu Q(u))=K \sqrt[\eta+r+1]{b^{1-\gamma}-(b-u)^{1-\gamma}}
$$

where

$$
K=\sqrt[n+r+1]{\mu \frac{\eta+r+1}{r(1-\gamma)}}
$$

By a routine calculation, one can show that (for $0<c \leqslant b$ )

$$
\begin{aligned}
\int_{0}^{c} \frac{1}{H^{-1}(\mu Q(s))} \mathrm{d} s & =\frac{1}{K} \int_{0}^{c} \frac{1}{\sqrt[\eta+r+1]{b^{1-\gamma}-(b-s)^{1-\gamma}}} \mathrm{d} s \\
& =\frac{\sqrt[\eta+r+1]{b^{\eta+r+\gamma}}}{K} \int_{0}^{c / b} \frac{1}{\sqrt[\eta+r+1]{1-(1-s)^{1-\gamma}}} \mathrm{d} s \\
& =\frac{\sqrt[\eta+r+1]{b^{\eta+r+\gamma}}}{(1-\gamma) K} \int_{0}^{1-(1-(c / b))^{1-\gamma}} \frac{\sqrt[1-\gamma]{(1-s)^{\gamma}}}{\sqrt[n+r+1]{s}} \mathrm{~d} s
\end{aligned}
$$

Now

$$
\int_{0}^{b} \frac{1}{H^{-1}(\mu Q(s))} \mathrm{d} s=\sqrt[\eta+r+1]{\frac{r b^{\eta+r+\gamma}}{\mu(\eta+r+1)(1-\gamma)^{\eta+r}}} \mathrm{~B}\left(\frac{\eta+r}{\eta+r+1}, \frac{1}{1-\gamma}\right)
$$

where $\mathrm{B}(\cdot, \cdot)$ is the beta function, and so Theorem 3.1 guarantees that the BVP (3.8), (1.2) has a solution $x$ satisfying (3.1) if $0<\gamma<1, r>\eta$ and $\mu \in\left(0, \mu_{*}\right)$ with

$$
\mu_{*}=\frac{r b^{\eta+r+\gamma}}{(\eta+r+1)(1-\gamma)^{\eta+r}}\left(\frac{1}{T} \mathrm{~B}\left(\frac{\eta+r}{\eta+r+1}, \frac{1}{1-\gamma}\right)\right)^{\eta+r+1} .
$$


Applying Theorem 3.2, BVP (3.8), (1.3) has a solution $x$ satisfying (3.7) if $\gamma \in(0, \infty)$, $r>\eta$ and $\mu \in\left(0, \mu_{0}\right)$ with

$$
\mu_{0}=\frac{r b^{\eta+r+\gamma}}{(\eta+r+1)(1-\gamma)^{\eta+r}}\left(\frac{1}{T} \int_{0}^{1-(1-(c / b))^{1-\gamma}} \frac{\sqrt[1-\gamma]{(1-s)^{\gamma}}}{\sqrt[\eta+r+1]{s}} \mathrm{~d} s\right)^{\eta+r+1} .
$$

Example 3.4. Consider the BVP

$$
\begin{gathered}
x^{\prime \prime}(t)=\mu\left(1-|x(t)|^{\gamma}\right)\left(\frac{1}{\left|x^{\prime}(t)\right|^{\eta}}-1\right), \\
x^{\prime}(0)=0, \quad x(1)=\frac{1}{2},
\end{gathered}
$$

where $\gamma \in(0, \infty)$ and $\eta \in(0,1)$. Assumptions $\left(H_{1}\right)-\left(H_{3}\right)$ are satisfied with $T=1$, $b=1 / 2, g(u)=u, \alpha(t)=1, \varepsilon=1-1 / 2^{\gamma}, \varepsilon_{0}=1 / \sqrt[n]{2}, \nu=1, q_{1}(x)=0, q_{2}(x)=1$, $\omega_{1}(y)=0$ and $\omega_{2}(y)=1 / y^{\eta}$. Hence

$$
Q(u)=\int_{0}^{u} \mathrm{~d} s=u, \quad H(u)=\int_{0}^{u} s^{\eta+1} \mathrm{~d} u=\frac{u^{\eta+2}}{\eta+2}
$$

and

$$
H^{-1}(\mu Q(u))=\sqrt[\eta+2]{(\eta+2) \mu u}
$$

Since

$$
\int_{0}^{1 / 2} \frac{1}{H^{-1}(\mu Q(s))} \mathrm{d} s=\int_{0}^{1 / 2} \frac{1}{\sqrt[\eta+2]{(\eta+2) \mu u}}=\frac{1}{\eta+1} \sqrt[\eta+2]{\frac{1}{\mu}\left(1+\frac{\eta}{2}\right)^{\eta+1}}
$$

Equation (2.12) (with $b=1 / 2$ and $T=1$ ) has the unique solution

$$
\mu_{*}=\frac{(\eta / 2+1)^{\eta+1}}{(\eta+1)^{\eta+2}} .
$$

Now Theorem 3.1 guarantees that BVP (3.9), (3.10) with $\mu \in\left(0, \mu_{*}\right)$ has a solution $x$ satisfying the inequalities $0<x(t) \leqslant 1 / 2$ and $0 \leqslant x^{\prime}(t) \leqslant 1$ for $t \in[0,1]$.

Acknowledgements. S.S. was supported by grant no. 201/01/1451 from the Grant Agency of the Czech Republic and by the Council of the Czech Government J14/98:153100011.

\section{References}

1. A. Granas, R. B. Guenther and J. W. Lee, Nonlinear boundary value problems for ordinary differential equations, Dissertationes Mathematicae, vol. 244 (Warsaw, 1985).

2. P. Kelevedjiev, Nonnegative solutions to some singular second-order boundary value problems, Nonlin. Analysis 36 (1999), 481-494.

3. J. Kong AND J. WANG, Multiple positive solutions for the one-dimensional $p$-Laplacian, Nonlin. Analysis 42 (2000), 1327-1333.

4. L. Kong AND B. G. ZhANG, Existence of nonnegative solutions for a class of singular boundary value problems, Dyn. Syst. Applic. 9 (2000), 435-444.

5. D. O'REgan, Existence of nonnegative solutions for a class of semi-positone problems, Dyn. Syst. Applic. 6 (1997), 217-230. 